\section{Immunohistochemical Study of CD68, CD3 and Bcl-2 and their Role in Progression and Prognosis of Head and Neck Squamous Cell Carcinoma}

\section{Abstract}

Head and neck squamous cell carcinoma is diagnosed at late stages. The prevalence of metastatic lesions directly correlates with poor patient outcome. The progression to metastatic disease requires changes in the tumor microenvironment. Within the microenvironment, infiltrating immune cells facilitate the spread of tumor cells. Tumor-infiltrating lymphocytes play an important role in the response of tumors to therapy. Tumor-associated macrophages are the main cellular component in stroma of many tumors. The goal of cancer treatment is to abolish cell proliferation and to induce aptoptosis. Apoptosis is a key mechanism of tumor cell elimination. The $\mathrm{Bcl} 2$ protein itself is a product of a proto-oncogene and has an antiapoptotic action. The study used immunohistochemical staining of CD68, CD3, $\mathrm{Bcl} 2$ to measure macrophages, lymphocytes and evaluate apoptosis in 25 included cases; 5 cases of non specific dermatitis, 5 normal skin and 15 head and neck squamous cell carcinoma (HNSCC) graded into 5 well, 5 moderately and 5 poorly differentiated. CD68+ve macrophages were significantly higher in moderately differentiated HNSCC than well differentiated tumors. Poorly differentiated showed significantly lower CD68+ve macrophages than moderately differentiated tumors. CD3 +ve lymphocytes were significantly higher in well differentiated followed by moderate and poorly differentiated. CD3+ve lymphocytes were significantly higher in cases of dermatitis than HNSCC cases. Poorly differentiated SCC showed higher Bcl 2 positivity followed by moderately differentiated. Well differentiated SCC showed the least Bcl2 positivity. This difference was significant. Our results showed tumor infiltrating macrophage and lymphocyte can predict the progression and prognosis of HNSCCs and the involvement of $\mathrm{BCl} 2$ in $\mathrm{HNSCC}$ tumorigenesis.

Keywords: Head and neck, SCC, Infiltrating macrophages, CD68, Lymphocytes, CD3, Apoptosis, BCL2, Stromal microenvironoment

\section{Duaa S Helal ${ }^{1}$ and Omneya M Wahba²

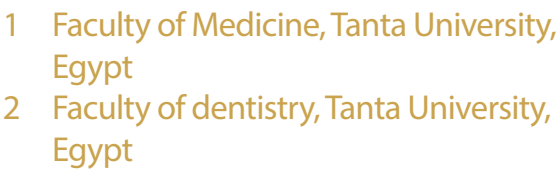

Corresponding author:

Duaa Samir Helal

Faculty of Medicine, Tanta University, Egypt झDuaahelal@yahoo.com

Tel: 201227130447

Citation: Helal DS, Wahba OM. Immunohistochemical Study of CD68, CD3 and $\mathrm{Bcl}-2$ and their Role in Progression and Prognosis of Head and Neck Squamous Cell Carcinoma. Arch Cancer Res. 2016, 4:1.

\section{Introduction}

Cancer has been considered a process in which some mutations in the oncogenes and tumor suppressor genes lead to the unlimited proliferation of neoplastic cells [1]. So, therapeutic strategies have been directed to target the process that occurs within the tumor cells [2].

Recent studies have concluded that for the effective treatment of cancer, its aggressiveness and progression must not only be considered to be cell autonomous, but also as a disease that involves complex multicellular interactions within the newly formed tissue and the tumor cells. The disease must be considered to be a systemic, complex, solid-tumor disease rather than a single disease. Therefore, the tumor microenvironment (TME) has been proposed as an important area of cancerous tissues within the tumor [3].

The TME include different cell types that affect the fate of cancer cells, the aggressiveness of the tumor and also the clinical outcome. TME cell population includes fibroblasts, myofibroblasts, smooth muscle cells, endothelial cells, leukocytes 
as neutrophils, basophils, mast cells, T and B cells and antigen presenting cells as macrophages and dendritic cells [4,5]. As the cancer cells proliferate, the surrounding TME is activated to produce continuous tumor-stromal interactions [6].

One of the main cellular components in the stroma of many cancers are tumor-associated macrophages (TAM) [7]. Macrophages have an important role in tumor progression due to their variable roles in promoting angiogenesis, expression of metalloproteinases and suppression of adaptive immunity [7-9].

TAMs have a pivotal role in tumor progression. They can perform cancer progression via controlling tumor angiogenesis, cancer invasion and migration. TAMs, which are often present in cancer, share many common criteria with the activated anti-inflammatory macrophages (M2) [10].

Macrophages can be classified into M1 macrophages (proinflammatory) and M2 macrophages (anti-inflammatory). M1 macrophages activate $\mathrm{T}$ helper cells; have an important role for eliminating pathogens and killing tumor cells. M2 macrophages play a role in tissue repair as they decrease the inflammatory reactions, promote angiogenesis and attract fibroblasts. M2 macrophages also have a weak tumoricidal action [11].

Tumor infiltrating lymphocytes (TILs) are important component in TME with an important role in inhibiting tumor proliferation, metastasis and may be used as an independent prognostic marker $[12,13]$. The percentages of infiltrating lymphocytes within the tumor cell have been reported to associate with good prognosis in different kind of tumors. CD3 antigen is a receptor glycoprotein present on all T lymphocytes [14,15].

The prognostic value of TILs in a tumor microenvironment is still unclear. In some researches, higher levels of CD3 TIL within the TME were associated with good prognosis as in oropharyngeal cancer [16]. But others showed low CD3 count had more better prognostic score in colon and cervical cancer $[17,18]$.

Transformation of cells from normal to cancer is a result of mechanisms that control cell mitosis and growth. Malignant cells escape away from the control of mitotic process. As mitotic process, death of cell (apoptosis), may be an active process which is also controlled via genetic mutations [12].

B-cell lymphoma/leukemia-2 (Bcl-2) oncogene was first discovered as the site of reciprocal translocation of chromosome 18 in lymphoma and located in a membrane associated protein. Over expression of $\mathrm{Bcl}-2$ in lymphoma, prostatic, lung and breast carcinoma concluded an important role for $\mathrm{Bcl}-2$ in the tumorgenesis $[13,14]$. The biological value of $\mathrm{Bcl}-2$ expression for the proliferation, progression and invasion of oral cancer is still unclear.

The current concept of malignant transformation of tissue is, downregulation of tumor suppressor genes, upregulation of oncogenes and changes in specific genes that regulate apoptosis, resulting in unregulated and uncontrolled proliferation of cells [15]. Increased the immunoexpression of $\mathrm{Bcl}-2$ leads to changes in apoptosis with failure of cell death. In proliferation of normal epithelial tissue, $\mathrm{Bcl}-2$ is expressed in basal cell layers, stem cell zones. Also, $\mathrm{Bcl}-2$ overexpression has been found in initial stage of malignant transformation of cells [16]. So, TME plays an important role in tumor progression.

The aim of the present study was to evaluate correlations between the $\mathrm{Bcl}-2$ expression, infiltrating macrophages (CD68) and CD3 tumour infiltrating lymphocytes TIL in non-specific dermatitis and different grades of squamous cell carcinoma [19-23].

\section{Materials and Methods}

\section{Tissue samples}

Squamous cell carcinoma biopsies from 25 patients were selected. 5 cases of non-specific chronic dermatitis and 5 cases of normal tissue. The clinical data and the anatomical description were collected from the archives of the Pathology department, Faculty of Medicine and Oral Pathology department, Faculty of Dentistry, Tanta University.

All included cases were stained with hematoxylin-eosin and reviewed in order to classify the cases according to the World Health Organization International Histological Classification of skin Tumours [24].

\section{Immunohistochemical staining}

Formalin-fixed and paraffin-embedded material was cut into $3-\mu \mathrm{m}$ thick sections, deparaffinized in xylene and rehydrated in ethanol and water. For antigen retrieval, tissue sections were boiled in citrate buffer, $\mathrm{pH}$ 6.0, for $40 \mathrm{~min}$ in a water bath at 9597으.

For immunohistochemical detection, antibodies to $\mathrm{CD} 68$ (monoclonal antibodies clone EBM11; Dako A/S; dilution 1:501:100; Code $\mathrm{M0718}$ ), antibodies to $\mathrm{Bcl}-2$ (monoclonal antibodies clone 124; Dako/Bcl-2; dilution 1:50-1:100; Code M0887) and antibodies to CD3 (monoclonal antibodies clone F7.2-38; Dako; dilution 1:25-1:50; Code M7254) were applied overnight using the EnVision TM System (Dako Corporation, Carpinteria, CA, USA). were used as the primary antibody for 30 minutes and was incubated in a moist chamber at room temperature $(24 \mathrm{~h})$ with a working dilution 1:50, followed by the application of secondary antibody (for 15 minutes), DAB (to produce brown staining), and Meyer's hematoxylin (for background staining). The samples were placed in phosphate buffer saline (PBS) after each mentioned step. Prostate gland and placenta was the positive control for maspin and CD34 respectively according to the manufacturer's instructions. The negative control was obtained by the replacement of primary antibody with PBS

\section{Evaluation of the immunostaining results}

Assessment of immunohistochemistry stained sections: Presence of brown colored reaction in the nucleus or cytoplasm was considered a positive reaction. The intensity of the immunostaining was classified as negative, weak or strong from three fields in a blinded analysis using a conventional diagnostic microscope and further image analysis was done with the Image J software (version 4.10.03, Nikon, Tokyo, Japan).

The assessment of the intensity of staining and its location was done.. A scale of 0 to ++ was used. The location of staining was assessed at both tissue and cellular level. Concerning the tissue 
level location, staining of lining cells, stellate reticulum and connective tissue were assessed. Further the areas were graded based on the staining intensity as, 0 - absence of staining, + weak positive staining, ++- strong positive staining. At cellular level, staining of cell membrane, cytoplasm and nucleus were assessed and graded as follows, $\mathrm{M}$ - membranous positivity, C cytoplasmic positivity, $\mathrm{N}$ - nuclear positivity and $\mathrm{B}$ - both.

\section{Statistical analysis}

The data were analyzed using version 20 of the SPSS. Qualitative data were compared using chi-squared statistics. Quantitative data were summarized using mean, standard deviation, and confidence interval and compared using Student's test and/or one way analysis of variance test. All $P$ values were determined, and statistical significance was based on a $P$ value of 0.05 .

\section{Results}

The study included 25 cases received as paraffin blocks. Serial sections were stained with $\mathrm{H} \& \mathrm{E}$ and the cases were histologically classified into 5 cases of non specific dermatitis, 5 cases of well differentiated SCC, 5 cases of moderately differentiated SCC and 5 poorly differentiated SCC. Five cases from normal skin were included as control group.

\section{Histopathological results}

Normal skin sections were included as control for the study Figure 1. Cases diagnosed as non specific dermatitis showed dense infiltration with chronic inflammatory cells in upper dermis and subcutis mainly perivascular. The overlying epidermis showed hyperkeratosis and acanthosis; 2 cases showed ulceration Figure 2. The infiltrate was composed mainly of lymphocytes admixed with plasma cells and macrophages. Squamous cell carcinoma showed nests of malignant epithelial cells arising from the epidermis and infiltrating the dermis Figures 3, 4 and 5. Malignant cells are large with pleomorphic nuclei. Intercellular

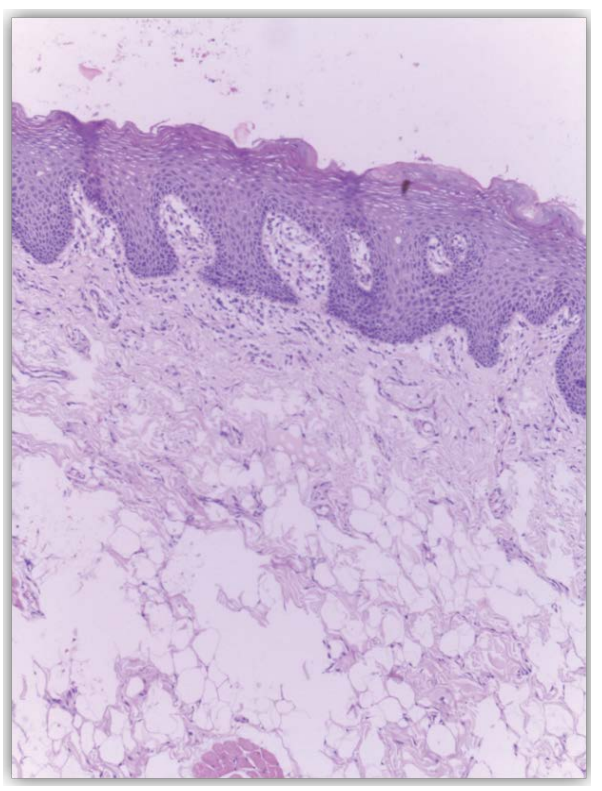

Figure 1 Section representive of normal skin (H\&E x 100).
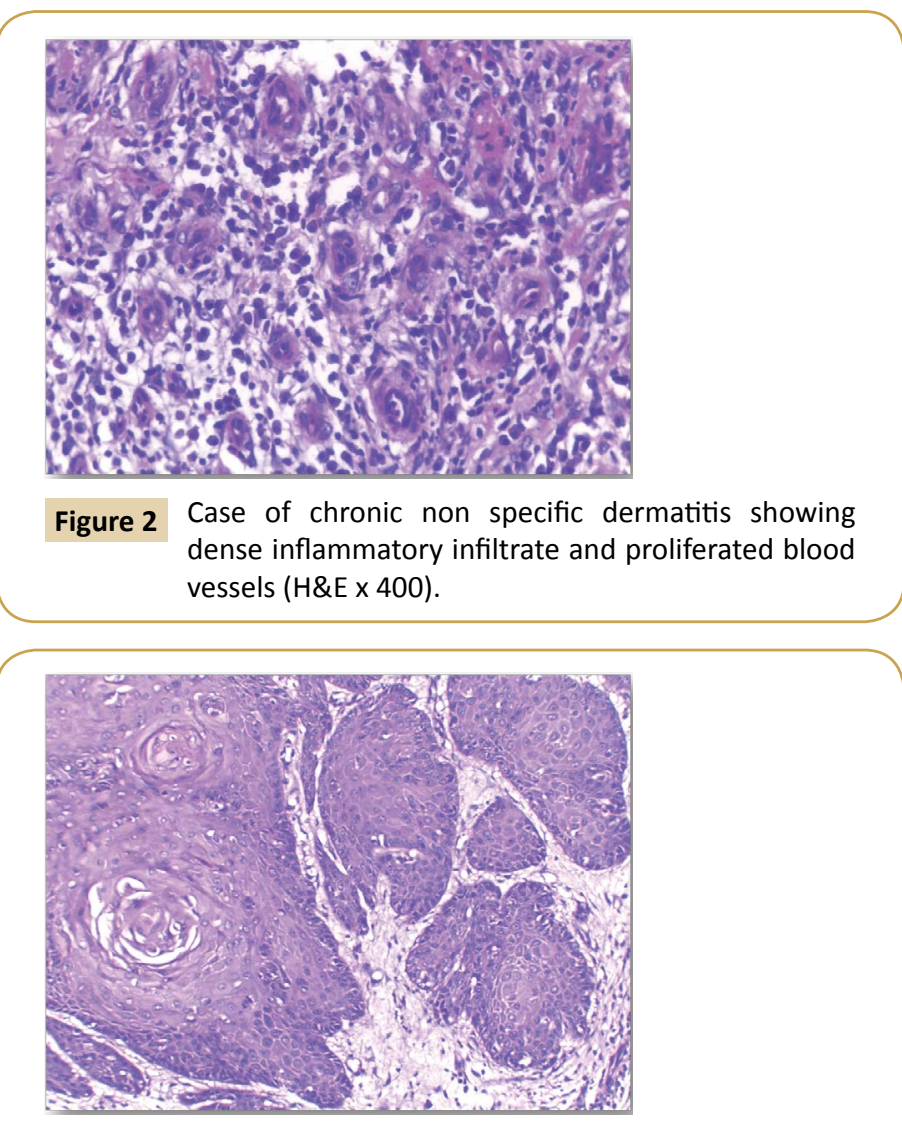

Figure 3 Well differentiated SCC showing infiltrating cell nests some of which showing keratin pearls. (H\&E x 200).

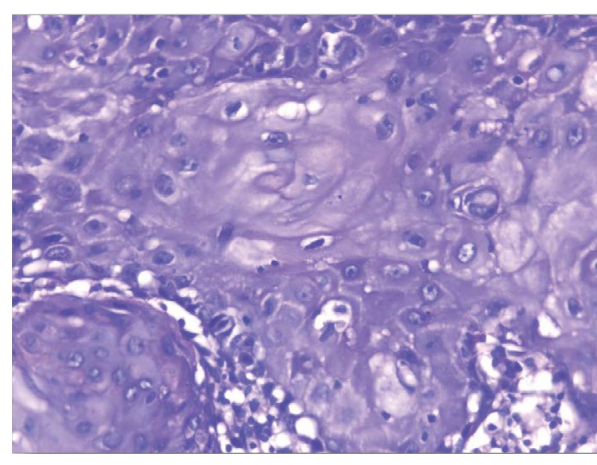

Figure 4 Moderately differentiated SCC with malignant squamous cells showing pleomorphism and moderate atypia (H\&E x 400).

bridges are present. Numerous atypical mitotic figures are noted. There are large amounts of keratin with keratin pearl formation depending on the differentiation of the tumour.

\section{Immunohistochemistry}

To examine the changes in cellular population (CD68 macrophages and CD3 lymphocytes) in tumor stroma, immunohistochemistry (IHC) was done in 4- $\mu \mathrm{m}$ paraffin-embedded sections. Bcl2 immunostain was used to evaluate apoptosis.

\section{Macrophages}

CD68-positive IMs were predominantly found in the subepithelial 


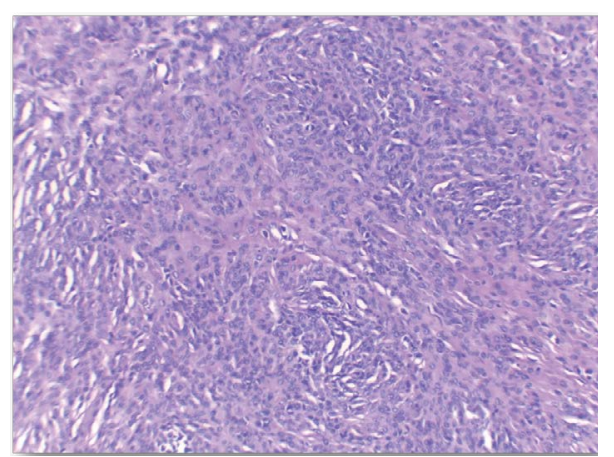

Figure 5 Poorly differentiated SCC with diffuse infiltration of dermis with malignant cells. (H\&E x 200).

connective tissue of normal and dermatitis samples and in the stromal connective tissue of SCC samples (Figure 6). The mean IMCs (macrophages/HPF) increased significantly from normal $(80.34 \pm 2.70)$ through dermatitis $(108.12 \pm 1.83)$, to SCC samples. The SCC samples were divided into three grades; well, moderate and poorly differentiated. CD 68 macrophages were significantly higher in cases of dermatitis than control sections of normal skin. In studied cases of SCC, the mean number of CD68 macrophages was higher in stroma of moderate $(102.05 \pm 1.82)$ and poorly $(101.50 \pm 2.09)$ differentiated cases than well $(112.44 \pm 2.49)$ differentiated SCC. The significant $p$ value is between each 2 groups Figures 7, 8 and 9. Correlation between the mean number of CD68 positive macrophages and the histological diagnosis is listed in Table 1 and illustrated in Graph 1.

\section{T Lymphocytes}

In this study, CD3-positive lymphocytes were observed in tumor stroma showing brown stained nuclei. CD3+ve lymphocytes were significantly denser in cases of non specific dermatitis (mean $108.12 \pm 1.83$ ). Sections from normal skin showed the lowest CD3 positive lymphocytes density (mean $80.34 \pm 2.70$ ). In studied cases of SCC; CD3 was significantly higher in well differentiated (mean $112.44 \pm 2.49)$ tumors followed by moderate $(102.05 \pm$ $1.82)$ and poorly $(101.50 \pm 2.09)$ differentiated cases. CD3 positive lymphocyte density was significantly higher in cases of dermatitis than SCC cases. The significant $p$ value is between each 2 groups Figures 10, 11 and 12. Correlation between the mean number of CD3 positive lymphocytes and the histological diagnosis is listed in Table $\mathbf{2}$ and illustrated in Graph $\mathbf{2}$.

\section{Bcl 2 immunostaining}

The mean number of $\mathbf{B c l} \mathbf{2}+\mathbf{v e}$ cells was significantly higher in control sections of normal skin $(89.35 \pm 2.55)$ than studied cases of non specific dermatitis ( $37.45 \pm 34.21)$. Within the SCC cases; poorly differentiated cases of SCC showed higher Bcl2 positivity (113.27 \pm 4.26$)$ followed by moderately differentiated tumors (93.08 \pm 2.71$)$. Well differentiated SCC (34.14 \pm 44.10$)$ showed the least $\mathrm{Bcl} 2$ positivity. This difference was found statistically significant. The significant $p$ value is between each 2 groups (Figures 13 - 17). Correlation between the mean number of $\mathrm{Bcl}-2$ positive cells and the histological diagnosis is listed in Table $\mathbf{3}$ and illustrated in Graph 3.
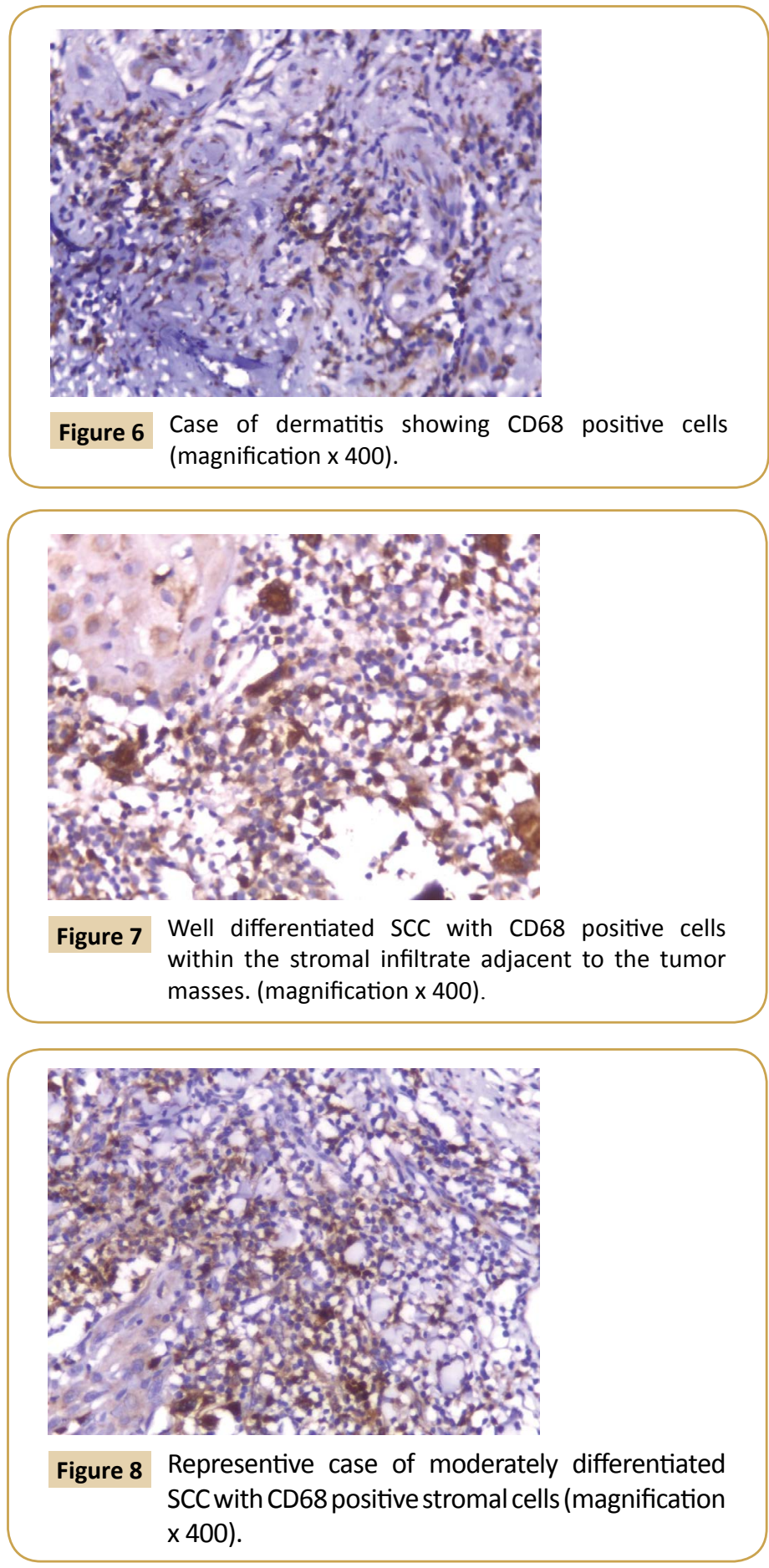

\section{Discussion}

About half of individuals diagnosed with head and neck squamous cell carcinoma (HNSCC) will survive for 5 years despite the significant advancements in surgery, radiation and chemotherapy only. Developing successful clinical approaches to therapy is difficult because HNSCC spreads by local and distant metastasis. The rich lymphatic drainage from the oral cavity and the highly invasive behaviour of HNSCC facilitate the early spread to regional lymph nodes. Better understanding of the underlying mechanisms regulating the invasive and metastatic behaviour of this malignancy is crucial [25]. 


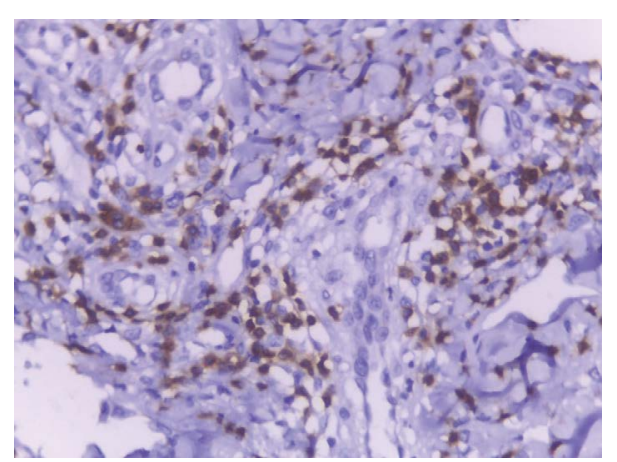

Figure 9 Poorly differentiated SCC with CD68 positive cells in perivascular stromal inflammatory infiltrate. (magnification $\times 400$ ).
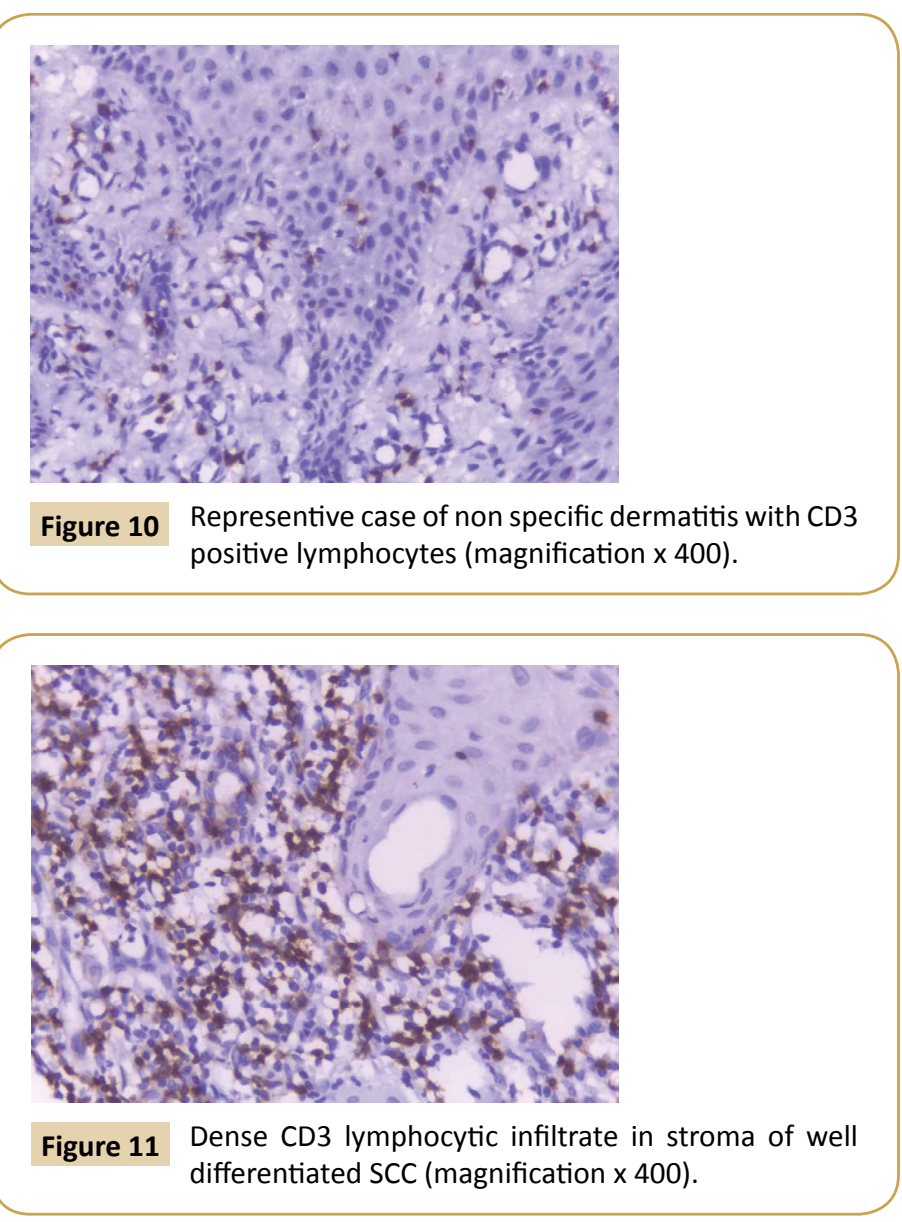

Head and neck squamous cell carcinoma is a complex tissue that contains tumor cells and the surrounding stroma, consisting of different types of mesenchymal cells and the extracellular matrix. Collectively they are called the tumor microenvironoment (TME). Recent studies have shown that TME has a more important effect on the growth and metastasis of HNSCC than was previously thought [2]. TME contains many cell types including fibroblasts, carcinoma associated fibroblasts, endothelial cells, pericytes, eosinophils, basophils and antigen presenting cells such as marcrophages and dendritic cells. CAFs, macrophages and dendritic cells in particular had shown an important role in previous studies [26].

Tumors are mixtures of both neoplastic and stromal cells. The

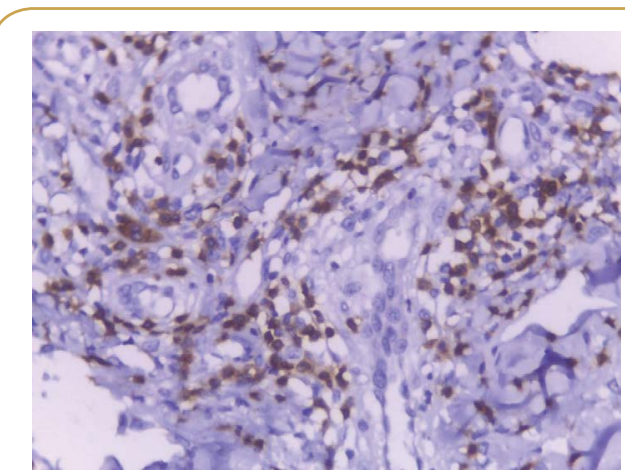

Figure 12 Poorly differentiated SCC with CD3 positive lymphocytes within the tumor stroma (magnification $\times 400$ ).

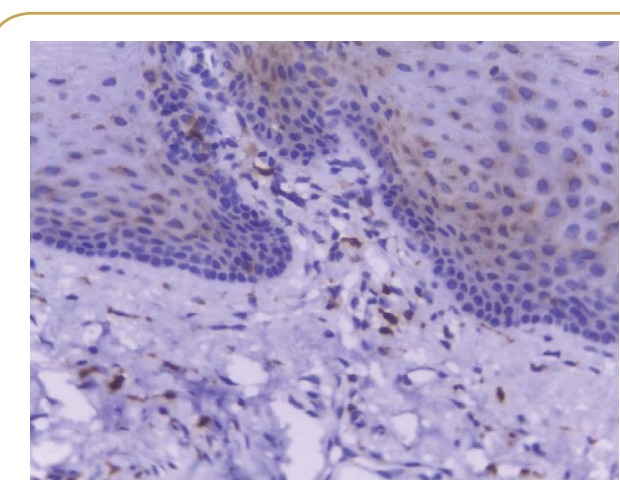

Figure 13 Representive case of normal skin section with minimal $\mathrm{Bcl}-2$ positive cells scattered within the dermis (magnification $\times$ 400).

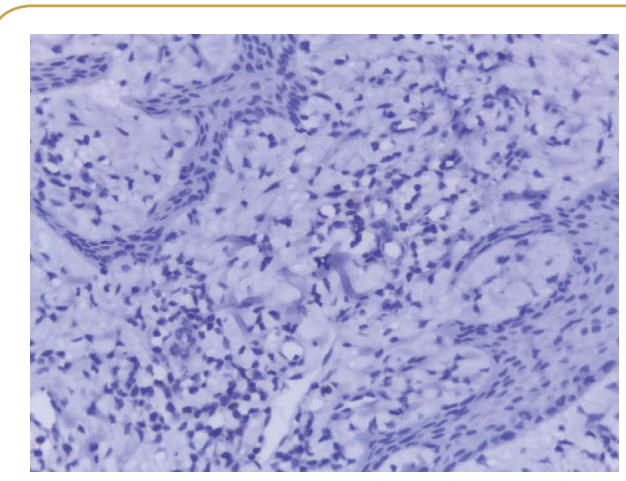

Figure 14 A case of non specific dermatitis showing complete negativity for $\mathrm{Bcl}-2$ immunostain. (magnification $\mathrm{x}$ 400).

occurrence and the growth of tumors require support from the stromal microenvironment surrounding the tumor and does not only depend on the factors intrinsic to the tumor. The complex interactions between tumor cells and the elements within the microenvironment play a pivotal role in cancer initiation, progression and invasion $[27,28]$.

The interaction between the host immune system and the neoplastic growth is acknowledged by the research that correlates tumor infiltrating lymphocytes (TILS) with outcomes of patients with solid tumors [29]. T cell function is important 
Table 1 Showing the mean values of CD68 in studied cases of normal, dermatitis and SCC cases.

\begin{tabular}{|c|c|c|c|c|c|}
\hline Mean & Normal skin & Dermatitis & Well differentiated SCC & Moderately differentiated SCC & $\begin{array}{l}\text { Poorly differentiated } \\
\text { SCC }\end{array}$ \\
\hline Range & $72.74-78.17$ & $103.59-109.67$ & $65.95-72.71$ & $108.77-125.13$ & $76.4-81.16$ \\
\hline Mean + SD & $75.45 \pm 2.15$ & $107.42 \pm 2.3$ & $69.29 \pm 2.58$ & $119.65 \pm 6.04$ & $78.57 \pm 1.94$ \\
\hline F test & \multicolumn{5}{|c|}{74.725} \\
\hline$P$ value & \multicolumn{5}{|c|}{$0.001^{*}$} \\
\hline
\end{tabular}

Table 2 Showing the mean values of CD3 lymphocytes in studied cases of normal, dermatitis and SCC cases.

\begin{tabular}{|c|c|c|c|c|c|}
\hline Mean & Normal skin & Dermatitis & Well differentiated SCC & $\begin{array}{c}\text { Moderately differentiated } \\
\text { SCC }\end{array}$ & $\begin{array}{c}\text { Poorly differentiated } \\
\text { SCC }\end{array}$ \\
\hline Range & & & & $99.44-104.16$ & $98.68-104.32$ \\
\hline Mean + SD & $86.72-83.46$ & $105.78-110.31$ & $109.78-116.41$ & $102.05 \pm 1.82$ & $101.50 \pm 2.09$ \\
\hline F test & & $108.12 \pm 1.83$ & $112.44 \pm 2.49$ & 85.824 & \\
\hline P value & & & $0.001^{*}$ & \\
\hline
\end{tabular}

Table 3 Showing the mean values of Bcl2 in studied cases of normal, dermatitis and SCC cases.

\begin{tabular}{|c|c|c|c|c|c|}
\hline Mean & Normal skin & Dermatitis & Well differentiated SCC & Moderately differentiated SCC & Poorly differentiated SCC \\
\hline Range & $86.21-92.79$ & $0.0-64.46$ & $0.0-87.64$ & $90.19-97.02$ & $108.12-118.47$ \\
\hline Mean + SD & $89.35 \pm 2.55$ & $37.45 \pm 34.21$ & $34.14 \pm 44.10$ & $93.08 \pm 2.71$ & $113.27 \pm 4.26$ \\
\hline F test & & & 11.645 & $0.001^{*}$ \\
\hline P value & & & 3 & \\
\hline
\end{tabular}

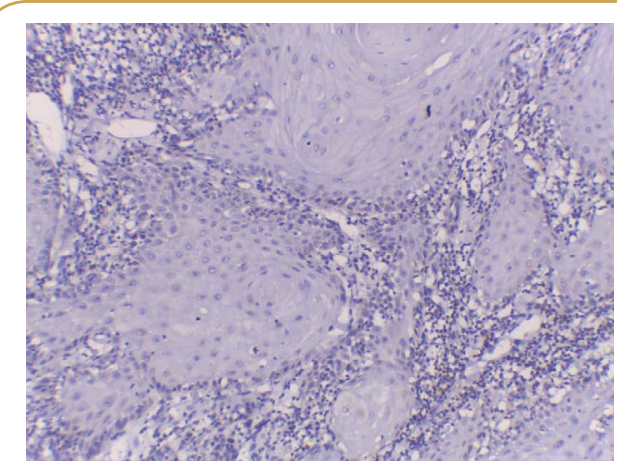

Figure 15 A case of well differentiated SCC showing complete negativity for Bcl-2 immunostain. (magnification $\times 100$ ).

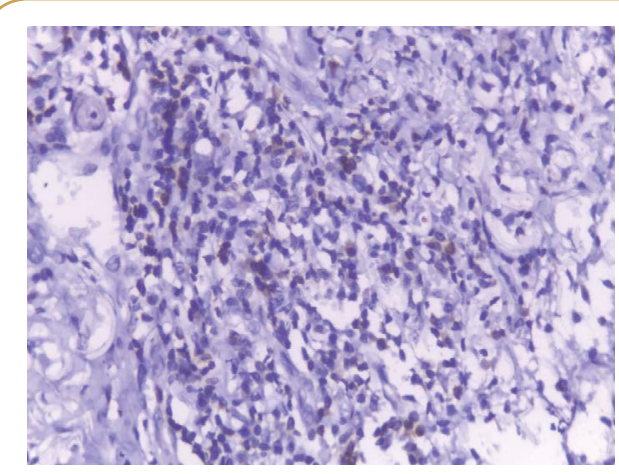

Figure 16 A case of moderately differentiated SCC showing positive Bcl-2 immunostain within the stromal infiltrate (magnification $x$ 400).

to the immunological control of SCCs. Patients on T cellimmunosuppressant medications were found more susceptible to SCCs; this finding suggests that T lymphocytes has a role in controlling these tumours. Organ transplant recipients receiving

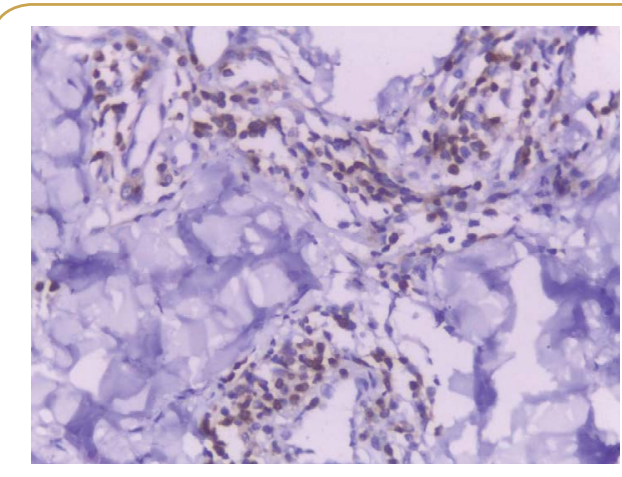

Figure 17 Poorly differentiated SCC with dense Bcl-2 positive cells within the stromal perivascular infiltrate.

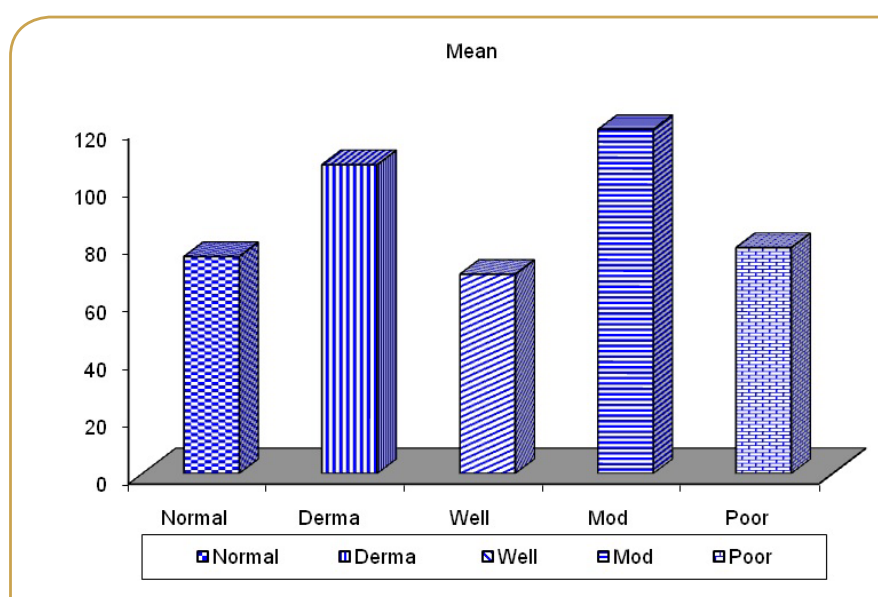

Graph 1 Showing the mean values of CD68 in studied cases of normal, dermatitis and SCC cases.

of medications that suppress $\mathrm{T}$ cell activity develop multiple high grade SCCs with poor prognosis and high recurrence [30]. Dysfunctional circulating and tumor-infiltrating $T$ cells were found 


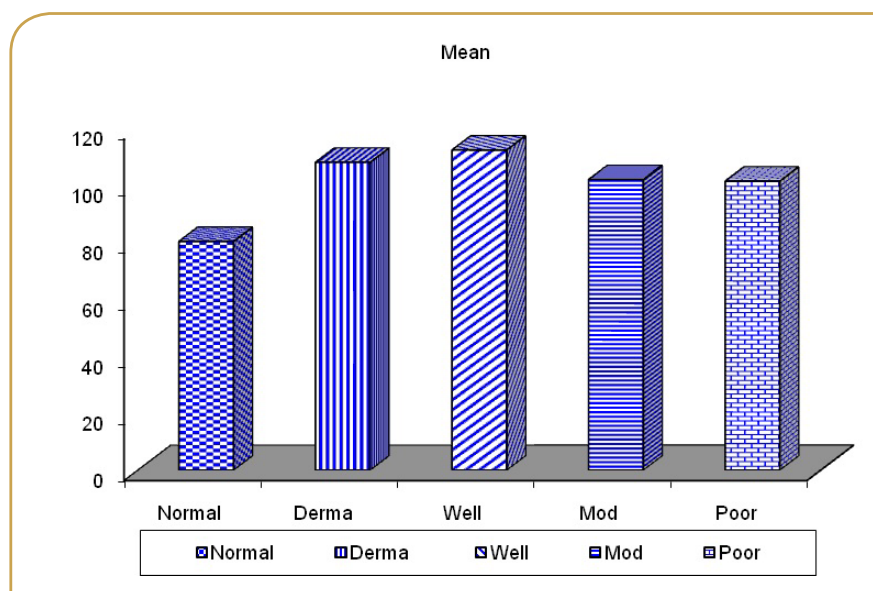

Graph 2 Showing the mean values of CD3 lymphocytes in studied cases of normal, dermatitis and SCC cases.

\section{Mesn}

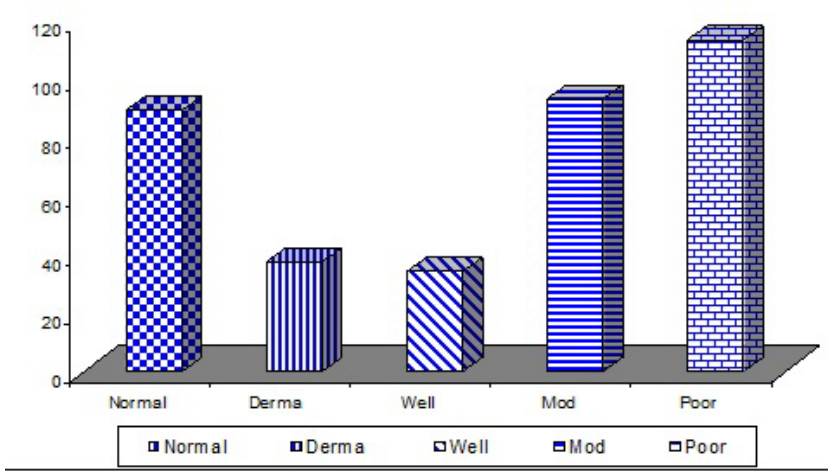

Graph 3 Showing the mean values of $\mathrm{Bcl} 2$ in studied cases of normal, dermatitis and SCC cases.

in HNSCC patients showing multiple defects in T-cell activation and function. CD3 is a pan-T cell marker and constitutes a protein complex involving four distinct chains that is integral part the T-cell receptor (TCR).

This study found a significant increase in the mean CD3+ve lymphocytes from normal (mean 76.72 - 83.46) through inflammatory lesions (mean 105.78 - 110.31) to SCC samples. A significantly increased CD3+ve lymphocytes was found in well differentiated SCC (mean 109.78 - 116.41) followed by moderately differentiated tumors (mean 99.44 - 104.16) and poorly differentiated tumors (mean 98.68 - 104.32). This finding is consistent with the concept that enhanced immunity have protective effect for cancer patients. This result was in agreement with Brandwein-Gensler et al., who demonstrated that strong lymphocytic response at stromal-tumoral interface in SCC is associated with improved outcome [31]. Balermpas et al., demonstrated that the high levels of CD3+ and CD8+ T cells is a good prognostic factor in SCC patients. Patients showed good prognosis and less metastatic tumors as compared with cases with low $\mathrm{CD} 3+$ and $\mathrm{CD} 8+\mathrm{T}$ cells [32]. These results are in line with previous studies of Gooden et al., whom concluded that heavy infiltration of HNSCC by CD3+ and CD8+ T lymphocytes is associated with good prognosis in patients affected by HNSCC as well as colorectal, lung, breast, ovarian, esophageal, renal and anal carcinomas [33] . In contrary, Denkert et al., studied the compartmental abundance of T lymphocytes in SCC and concluded that the prognostic value of tumor infiltrating lymphocytes differs according to their location within the tumor. High levels of CD3+ $T$ lymphocytes in the area near malignant cells had an improved disease outcome whereas CD3+ T-cell infiltration of the tumor stroma or periphery had no effect on prognosis [34].

Brandwein et al., examined lymphocyte infiltration into over 200 cases of oral SCCs. They concluded that a weak lymphocytic reaction at the tumor-stromal interface was associated with increased recurrence and decreased survival [31].

Sewell and colleagues analyzed CD3+ T lymphocytes in 48 different oropharyngeal squamous cell cancers cells by immunohistochemistry and concluded that tumors that were high in CD3 lymphocyte density showed decreased metastasis compared to those that were CD3 low. Interestingly, the largest tumors had the lowest CD3 levels [35].

On the contrary to the previous studies Uppaluri et al., studied over 35 squamous cell carcinomas and found no relation between $\mathrm{CD} 3+, \mathrm{CD} 4+$ or CD8+ T cell infiltration and survival [29].

Macrophages are important cells for the innate immunity. M1 macrophages are activated by bacterial products or lipopolysaccharides; they kill microorganisms and cancer cells. M2 macrophages are activated by cytokines and have tumorpromoting activities. The tumoral infiltrating macrophages IM are considered to be a kind of $\mathrm{M} 2$ macrophage [36].

Tumour associated macrophages (TAMs) represent a main component of the infiltrating leukocytes in tumours. TAMs are present at higher levels in SCC and control angiogenesis during progression of the tumour [37]. High TAM infiltration in cases of primary HNSCC is highly related to lymph node metastasis, extracapsular spread and advanced stages [38].

Infiltrating macrophage count (IMC) has a critical role in the prognosis of many human cancers. Increased IMC in cancer stromal tissues is associated with tumor progression, metastasis, and recurrence as well as a decreased survival in patients with a variety of cancers. Elevated IMC is also associated with increased microvessel density, lymph node involvement,tumor size, and extracapsular lymph node spread, advanced clinical stage, and poor survivalin patients with oral and/or oropharyngeal SCCs. The influence of IMC on the transformation from normal epithelium into precancer and further into an SCC was unclear [39].

In this study, we used immunohistochemistry to measure the IMC in tissue sections of normal skin, dermatitis, and SCC. We tried to find whether the IMC had a significant influence on the transformation of normal squamous epithelium and inflammatory skin lesions into SCC and on the progression and prognosis of SCCs.

In the present study CD68 macrophages were found significantly increasing in number from normal to dermatitis to SCC cases. This increase in macrophage density may be due to the increase of CCL2 (monocyte chemoattractant protein-1) and IL-6 which induce M2-macrophage differentiation; they increase in many 
cancer types, including oral squamous cell carcinoma whereas normal epithelial cells has lower levels of these cytokines [40]. These results coincided with Lu et al., who found a significant and gradual increase in the mean infiltrating macrophages from normal through mild, moderate, and severe dermatitis to SCC samples. These findings suggest that the increased infiltrating macrophages may correlate with the transformation of normal squamous epithelium into a precancer and further into an SCC [41].

Within the malignant cases, CD68 macrophages were significantly higher in moderately differentiated tumors than well differentiated tumors, this increase in CD68 macrophages with increased tumor dediffrentiation could be explained by increased levels of various cytokines and chemokines that support macrophage survival and differentiation into the $\mathrm{M} 2$ phenotype in higher SCC grades and the tumor microenvironment [42]. These results were in agreement with Liu et al., who showed a significant correlation between the higher IMC and larger tumor size, lymph node metastasis, and poor survival [43]. Also Marcus et al., showed a significant association of higher IMC with lymph node metastasis, local spread and advanced clinical stage in a series of SCCs [38].

The present study found a significantly lower CD68 macrophage density in poorly differentiated tumors than moderately differentiated SCC. This decrease could be due to dedifferentiating M1 macrophages during the progression of the high-grade tumors [44].

On the contrary to the previous results Mori et al., found no correlation between the number of CD68+ cells and the tumor grade but reported that $\mathrm{CD} 163+\mathrm{M} 2$ macrophages, in OSCC specimens, were significantly increased based on the pathological grade. Also a study by Boas et al., and ElRouby, found TAM largely present in all studied SCC tumors and concluded that IMD was not different among HNSCCs with different histological grades $[29,45]$. This difference was explained by the mixed cell populations of macrophages including $\mathrm{M} 1, \mathrm{M} 2$ and undifferentiated monocytes/ macrophages which are all positive for CD68 (pan macrophage marker), these types of macrophages play different roles in the development and progression of HNSCC [46].

Because IMs may play a pivotal role in the progression of human cancers, targeting IMs may be another therapeutic strategy for human cancers. Therapies to target IMs and decrease their density or IM-associated angiogenesis in tumor tissues found to impair cervical carcinogenesis, decrease melanoma growth, and inhibit breast cancer progression [47]

Chronic inflammation increases the risk of cancer, and many cancers arise from sites of chronic inflammation [48]. For example, Helicobacter pylori infection is associated with formation of gastric cancer; hepatocellular carcinomas arise from livers with chronic hepatitis B or C; and colon cancers derive from colons with chronic colitis. HNSCCs often derive from OED lesions [49]. This study found a significant and gradual increase in the mean IMCs from normal epithelium into precancer and further into SCC. These findings suggest that the increased IMC may correlate with the transformation of normal epithelium with chronic inflammation into SCC.

Apoptosis (programmed cell death) is essential during development and in the maintenance of tissue homeostasis, and serves to delete damaged or redundant cells. Disruption of the normal mechanisms for apoptosis can result in a number of pathological conditions. Apoptosis is also of important significance in the pathogenesis of cancer. The survival and propagation of cells with damaged DNA may contribute to the development of neoplasia and metastasis [50]. Several studies have indicated that the capacity of a tumour to undergo apoptosis may influence its behaviour and response to therapy. Apoptosis is possibly the principal mechanism by which irradiation and chemotherapy kill cancer cells. Evidence suggests the role of $\mathrm{Bcl}$ 2 in chemoresistance [51].

Dysregulation of $\mathrm{Bcl}-2$ expression, which results in abnormal cell growth, certainly contributes to the development of some tumors. Abnormally high levels of $\mathrm{Bcl}-2$ are predicted to occur in more than half of all human cancers [52]. Elevated expression of $\mathrm{Bcl}-2$ in some tumors is often associated with shorter survival time and generally poorer clinical outcomes. Furthermore, overexpression of $\mathrm{Bcl}-2$ is associated with enhanced invasion and metastasis [53].

The importance of $\mathrm{Bcl}-2$ overexpression in cancer biology is revealed by it high frequency of occurrence in many human malignancies. The biological significance of $\mathrm{Bcl}-2$ over-expression in tumours has varied widely in different reports. It appears that the significance of $\mathrm{Bcl}-2$ over-expression may depend on the biology of the individual tumour [54]. Alterations in the expression level of apoptosis-associated proteins have been reported in several cancers, including oral cancer [55].

In the present work BCL2expression was detected in all studied cases of normal skin, dermatitis and SCC. The BCL2 expression was significantly correlated with histologic tumor grades. It was higher in poorly differentiated tumors followed by moderately differentiated tumors while well differentiated tumors showed the least BCL2 expression. These results were in agreement with Zuo et al., who showed ectopic overexpression of $\mathrm{Bcl}-2$ in the human squamous carcinoma cell line HSC-3 enhanced tumorigenicity and experimental pulmonary metastasis [56]. Also, Cor et al., who reported that $\mathrm{Bcl}-2$ was undetectable in well differentiated tumours but it appeared to be up-regulated in one moderately and two poorly differentiated tumours [57]. On the contrary to previous reports Coutinho-Camillo et al., found that expression of $\mathrm{Bcl}-2$ in HNSCC was not significantly associated with any of the clinicopathological parameters. This difference in results could be explained by the large number of cases (229) included in their study [58].

\section{Conclusion}

The present study revealed a cellular response in the HNSCC tumor microenvironment including CD3 T lymphocyte and CD68 macrophages infiltration. Those cellular responses are required to continue supporting SCC cell growth and progression through forming a supportive microenvironoment. CD3 T lymphocytes were significantly correlated with the tumor grades which may point to the importance of the combination of CRT with targeted immunotherapies that activate T cells in HNSCC patients with low levels of $\mathrm{CD} 3+$ tumor infiltrating lymphocytes to enhance treatment outcomes. 
CD68 macrophages were significantly associated with the transformation from normal epithelium through inflammation and further SCC. IMC can be used as a predictor for the progression and prognosis of SCC. Apoptotic marker Bcl2 was significantly correlated with worse tumor grades. These results show the possible role of $\mathrm{Bcl} 2$ in progression and treatment of HNSCC. Further studies are required to determine the exact role of these cells in influencing the growth, invasion, and metastasis of SCC. To seek novel therapeutic interventions that targets the tumor stromal components. 


\section{References}

1 Kenny PA, Lee GY and Bissell MJ (2007) Targeting the tumor microenvironment. Front Biosci 12: 3468-3474.

2 Koontongkaew S (2013) The tumor microenvironment contribution to development, growth, invasion and metastasis of head and necksquamous cell carcinomas. J Cancer 4: 66-83.

3 Liotta LA, Kohn EC (2001) The microenvironment of the tumour-host interface. Nature 411: 375-379.

4 Rivera C, Venegas B (2009) Histological and molecular aspects of oral squamous cell carcinoma (Review). Oncol Lett 8: 7-11.

5 Pollard JW (2004) Tumor educated macrophages promote tumour progression and metastasis. J Nat Res Cancer 4: 71-78.

6 Pietras K, Ostman A (2010) Hallmarks of cancer: interactions with the tumorstroma. Exp Cell Res 316: 1324-1331.

7 Mantovani A, Sica A (2010) Macrophages, innate immunity and cancer: balance, tolerance, and diversity. CurrOpinImmunol 22: 231-237.

8 Mantovani A, Sozzani S, Locati M, Allavena P, Sica A (2002) Macrophage polarization: tumor-associated macrophages as a paradigm for polarized M2 mononuclear phagocytes. Trends Immunol 23: 549-555.

9 Coussens LM, Werb Z (2002) Inflammation and cancer. Nature 420: 860-867.

10 Sica A, Schioppe T, Mantovani A, Allavena P (2006) Tumor-associated macrophages are a distinct $\mathrm{M} 2$ polarised population promoting tumour progression: potential targets of anti- cancer therapy. Eur J Cancer 42: 717-727.

11 Gordon S (2003) Alternative activation of macrophages. Nat Rev Immunol 3: 23-35.

12 Galon J, Costes A, Sanchez-Cabo F, Kirilovsky A, Mlecnik B, et al. (2006) Type, density, and location of immune cells within human colorectal tumors predict clinical outcome. Science 313: 1960-1964.

13 Nakano O, Sato M, Naito Y, Suzuki K, Orikasa S, et al. (2001) Proliferative activity of intratumoral CD8(+) T-lymphocytes as a prognostic factor in human renal cell carcinoma: clinicopathologic demonstration of antitumor immunity. Cancer Res 61: 5132-5136.

14 Black MM, Speer FD, Opler SR (1956) Structural representations of tumor-host relationships in mammary carcinoma; biologic and prognostic significance. Am J ClinPathol 26: 250-265.

15 Clemente CG, Mihm MC, Bufalino R, Zurrida S, Collini P, et al. (1996) Prognostic value of tumor infiltrating lymphocytes in the vertical growth phase of primary cutaneous melanoma. Cancer 77: 1303-1310.

16 Rajjoub S, Basha SR, Einhorn E, Cohen MC, Marvel DM, et al. (2007) Prognostic significance of tumor-infiltrating lymphocytes in oropharyngeal cancer. Ear Nose Throat J 86: 506-511.

17 Sinicrope FA, Rego RL, Ansell SM, Knutson KL, Foster NR, et al. (2009) Intraepithelial effector (CD3+)/regulatory (FoxP3+) T-cell ratio predicts a clinical outcome of human colon carcinoma. Gastroenterology 137: 1270-1279.

18 Ancuta E, Ancuţa C, Zugun-Eloae F, lordache C, Chirieac R, et al. (2009) Predictive value of cellular immune response in cervical cancer. Rom J MorpholEmbryol 50: 651-655.

19 Kuropkat C, Venkatesan TK, Caldarelli DD, Panje WR, HutchunPreisler HD, et al. (2002) Abnormalities of mole- cular regulators of proliferation and apoptosis in carcinoma of the oral cavity and oropharynx. Auris Nasus Larynx 29: 165-174.
20 Ben-Azra JM, Kornstein MJ, Grimes MM, Krystal G (1994) Small cell carcinomas of the lung express the Bcl-2 protein. Am J Pathol 145 : 1036-1040.

21 Lipponen P, Pietiainen T, Kosma VM, Aaltoma S, Eskelinen M, et al. (1995) Apoptosis suppressing protein Bcl-2 is expressed in welldifferentiated breast carcinomas with favorable prognosis. J Pathol 177: 49-55.

22 Kumar V, Abbas KA, Fausto N, Aster J, Robbins, et al. (2005) 7th edn WB Saunders: Pathologic Basis of Disease 270-340.

23 Loro LL, Vintermyr GK, Johannessen AC (2003) Cell death regulation in oral squamous cell carcinoma: methodological considerations and clinical significance. J Oral Pathol Med 32: 125-138.

24 Gale N, Pilch BZ, Sidransky D, Westra WH, Califano J (2005) Tumours of the oral cavity and oropharynx (epithelial precursor lesions) IARC Scientific Publications 181-190.

25 Barnes L, Eveson JW, Reichart P, Sidransky D,. World Health Organization classification of tumours.Pathology and genetics of head and neck tumours. Lyon: International Agency for Research on Cancer; IARC Scientific Publications 177-179.

26 Albini A, Sporn MB (2007) The tumour microenvironment as a target for chemoprevention. Nat Rev Cancer 7: 139-147.

27 Meng W, Wu Y, He X, Liu C, Gao Q, et al. (2014) A Systems Biology Approach Identifies Effective Tumor Stroma Common Targets for Oral Squamous Cell Carcinoma Cancer Res 74: 2306-2315.

28 Bagley RG (2010) The Tumor microenvironment. First edition. New York: Springer.

29 Uppaluri R, Dunn GP, Lewis JS (2008) Focus on TILs: prognostic significance of tumor infiltrating lymphocytes in head and neck cancers. Cancer Immun 8: 16.

30 Clark RA, Huang SJ, Murphy GF, Mollet IG, Hijnen D, et al. (2008) Human squamous cell carcinomas evade the immune response by down-regulation of vascular $E$ selectin and recruitment of regulatory T cells. The Journal of experimental medicine 205: 2221-2234.

31 Brandwein-Gensler M, Teixeira MS, Lewis CM, Lee B, Rolnitzky JJ, et al. (2005) Oral squamous cell carci-noma: histologic risk assessment, but not margin status, is strongly predictive of local disease-free and overall survival. Am J Surg Pathol 29: 167-78.

32 Balermpas P, Rödel F, Weiss C, Rödel C, et al. (2014) Tumor infiltrating lymphocytes favor the response to chemoradiotherapy of head and neck cancer, Oncolmmunology 3:1.

33 Gooden MJ, Bock GH, Leffers N, Daemen T, Nijman HW (2011) The prognostic influence of tumour infiltrating lymphocytes in cancer: a systematic review with meta-analysis. Br J Cancer 105: 93-103.

34 Denkert C, Loibl S, Noske A, Roller M, Müller BM, et al. (2010) Tumorassociated lymphocytes as an independent predictor of response to neoadjuvant chemotherapy in breast cancer. J Clin Oncol 28: 105-113.

35 Rajjoub S, Basha SR, Einhorn E, Cohen MC, Marvel DM, et al. (2000) Prognostic significance of tumor-infiltrating lymphocytes in oropharyngeal cancer. Ear Nose Throat J 86: 506-511.

36 Mantovani A, Sica A (2010) Macrophages, innate immunity and cancer: balance, tolerance, and diversity. Curr Opin Immunol 22: 231-237.

37 El-Rouby DH (2010) Association of macrophages with angiogenesis in oral verrucous and squamous cell carcinomas. J Oral Pathol Med 39: 559-564. 
38 Marcus B, Arenberg D, Lee J (2004) Prognostic factors in oral cavity and oropharyngeal squamous cell carcinoma. The impact of tumorassociated macrophages. Cancer 101: 2779-2787.

39 Linde N, Lederle W (2010) "Vascular endothelial growth factorinduced skin carcinogenesis depends on recruitment and alternative activation of macrophages.The Journal of Pathology 227: 17-28.

40 Roca H, Varsos ZS, Sud S, Craig MJ, Ying C, et al (2009) CCL2 and interleukin-6 promote survival of human $\mathrm{CD} 11 \mathrm{~b}+$ peripheral blood mononuclear cells and induce M2-type macrophage polarization. J Biol Chem 284: 34342-34354.

41 Lu CF, Huang CS, Tjiu JW, Chiang CP (2010) Infiltrating macrophage count: a significant predictor for the progression and prognosis of oral squamous cell carcinomas in Taiwan. Head Neck 32: 18-25.

42 Li C, Shintani S, Terakado N, Nakashiro K, Hamakawa H (2002) Infiltration of tumor associated macrophages in human oral squamous cell carcinoma. Oncol Rep 9: 1219- 1223

43 Liu SY, Chang LC, Pan LF, Hung YJ, Lee CH, et al. (2008) Clinicopathologic significance of tumor cell-lined vessel and microenvironment in oral squamous cell carcinoma. Oral Oncol 44: 277-285.

44 Mori K, Hiroi M, Shimada J, Ohmori Y (2011) Infiltration of M2 Tumor-Associated Macrophages in Oral Squamous Cell Carcinoma Correlates with Tumor Malignancy. Cancers 3: 3726-3739.

45 Boas D, Takiya M, Gurgel C, Carbal G, Santos, et al. (2013) TumorInfiltrating Macrophage and Microvessel Density in Oral Squamous Cell Carcinoma. Brazilian Dental Journal 3: 194-199.

46 Komohara Y, Hasita H, Ohnishi K, Fujiwara Y, Suzu S, et al. (2011) Macrophage infiltration and its prognostic relevance in clear cell renal cell carcinoma. Cancer Sci 102: 1424-1431.

47 Moussai D, Mitsui H, Pettersen JS (2011) The human cutaneous squamous cell carcinoma microenvironment is characterized by increased lymphatic density and enhanced expression of macrophage-derived VEGF-C. J Invest Dermatol 131: 229-236.
48 Balkwill F, Charles KA, Mantovani A (2005) Smoldering and polarized inflammation in the initiation and promotion of malignant disease. Cancer Cell 7: 211-217

49 Grivennikov, Sergei I, Florian RG, Michael K (2010) "Immunity, Inflammation, and Cancer". Cell 140: 883-899.

50 Hengartner MO (2000) The biochemistry of apoptosis. Nature 407: 770-776.

51 Yip KW, Reed JC (2008) Bcl-2 family proteins and cancer. Oncogene 27: 6398-6406.

52 Xie L, Qin W, Li J (2007) BNIPL-2 promotes the invasion and metastasis of human hepatocellular carcinoma cells. Oncol Rep 17: 605-610.

53 S Mena, M Benlloch, A Ortega (2007) Bcl-2 and glutathione depletion sensitizes B16 melanoma to combination therapy and eliminates metastatic disease. Clin Cancer Res 13: 2658-2666.

54 Condon LT, Ashman JN, Ell SR, Stafford ND, Greenman J, et al. (2002) Overexpression of $\mathrm{Bcl}-2$ in squamous cell carcinoma of the larynx: a marker of radioresistence. Int J Cancer 100: 472-475.

55 Loro LL, Vintermyr OK, Johannessen AC (2003) Cell death regulation in oral squamous cell carcinoma: methodological considerations and clinical significance. J Oral Pathol Med 32: 125-138

56 Jianhong Z, Tohru I, Shadi B, Zhiqiang $\mathrm{X}$, Joseph $\mathrm{OH}$, et al. (2010) Kramer: Angiogenesis, Metastasis, and the Cellular Microenvironment:Bcl-2 Overexpression Induces a Partial Epithelial to Mesenchymal Transition and Promotes Squamous Carcinoma Cell Invasion and Metastasis Mol Cancer Res 8: 170-182.

57 Cör A, Pižem J, Gale N (2002) The expression of bcl-2 and pro-caspase 3 in head and neck squamous cell carcinoma zdrav vestn 71: 39-43.

58 Coutinho-Camillo CM, Lourenc SV, Nishimoto IN, Kowalski LP (2010) Expression of Bcl-2 family proteins and association with clinicopathological characteristics of oral squamous cell carcinoma, Histopathology 57: 304-316. 